\title{
In vitro Comparison of Adipogenic Differentiation in Human Adipose-Derived Stem Cells Cultured with Collagen Gel and Platelet-Rich Fibrin
}

\author{
${ }^{1}$ Department of Oral and Maxillofacial Surgery, AB Shetty Memorial \\ Institute of Dental Sciences (ABSMIDS), Nitte University (Deemed \\ to be University), Deralakatte-575018, Mangaluru, India \\ 2 Nitte University Centre for Stem Cell Research and Regenerative \\ Medicine, K. S. Hegde Medical Academy, Nitte (Deemed to be \\ University), Deralakatte-575018, Mangaluru, India \\ ${ }^{3}$ Department of Plastic Surgery, K. S. Hegde Medical Academy, \\ Nitte (Deemed to be University), Deralakatte-575018, Mangaluru, \\ India
}

Pallavi Priyadarshini ${ }^{1}$ Soumi Samuel ${ }^{1}$, Basan Gowda Kurkalli² Chethan Kumar ${ }^{2}$

Basavarajappa Mohana Kumar ${ }^{2}$ Nikhil Shetty ${ }^{3}$ Veena Shetty ${ }^{2}$ Karthik Vishwanath ${ }^{3}$

Indian J Plast Surg 2021;54:278-283.

\begin{abstract}
Address for Correspondence Dr. Soumi Samuel, MDS, PhD, Department of Oral and Maxillofacial Surgery, AB Shetty Memorial Institute of Dental Sciences (ABSMIDS), Nitte University (Deemed to be University), Deralakatte-575018, Mangaluru, India (e-mail: soumisamuel@gmail.com).
\end{abstract}

\author{
Abstract \\ Keywords \\ - Adipose-derived stem \\ cells \\ - Collagen \\ - Platelet-rich fibrin \\ - Adipogenic \\ differentiation \\ - In vitro
}

Background: Adipose-derived stem cells (ADSCs) are the most preferred cell type, based on their phenotypic characteristics, plasticity, and favorable immunological properties for applications in soft-tissue augmentation. Hence, the present in vitro study was aimed to evaluate the adipogenic differentiation potential of human ADSCs upon culturing individually with collagen gel and platelet-rich fibrin (PRF).

Materials and methods: The collected lipoaspirate was used for establishing ADSCs using enzymatic digestion method. Then, the cells were analyzed for their morphology, viability, proliferation rate, population doubling time (PDT), colony-forming ability, cell surface markers expression, and osteogenic differentiation as biological properties. Further, ADSCs were evaluated for their adipogenicity using induction media alone, and by culturing with collagen gel and PRF individually for prospective tissue augmentation.

Results: ADSCs were successfully established in vitro and exhibited a fibroblast-like morphology throughout the culture period. Cells had higher viability, proliferation potential and showed their ability to form colonies. The positive expression of cell surface markers and osteogenic ability confirmed the potency of ADSCs. The ADSCs cultured on collagen gel and PRF, individually, showed higher number of differentiated adipocytes than ADSCs grown with adipogenic induction medium alone.

Conclusion: The extent of lipid accumulation by ADSCs was slightly higher when cultured on collagen gel than on PRF. Additional experiments are required to confirm better suitability of scaffold materials for soft-tissue regeneration. published online August 26, 2021
DOI https://doi.org/

$10.1055 / \mathrm{s}-0041-1733810$ ISSN 0970-0358
(C) 2021. Association of Plastic Surgeons of India.

This is an open access article published by Thieme under the terms of the Creative Commons Attribution-NonDerivative-NonCommercial-License, permitting copying and reproduction so long as the original work is given appropriate credit. Contents may not be used for commercial purposes, or adapted, remixed, transformed or built upon. (https://creativecommons.org/licenses/by-nc-nd/4.0/).

Thieme Medical and Scientific Publishers Pvt. Ltd. A-12, 2nd Floor, Sector 2, Noida-201301 UP, India 


\section{Introduction}

The replacement of soft-tissue components is necessary in various clinical conditions, such as breast and facial reconstruction, rectification of congenital deformities, or tumor resection. ${ }^{1}$ Combining functional rehabilitation with anatomic restoration poses a challenge in reconstructive surgery, which at present is achieved by selecting tissue form other regions of the body. Logically, multipotent stem cells offer the best option for multifaceted therapy to regenerate complex dermal tissue. Particularly, mesenchymal stem/stromal cells (MSCs) are enabled with features of self-renewal and multilineage differentiation. They have been investigated using different adult tissue sources. ${ }^{2}$ Adipose tissue as one of the main sources offers several advantages due to its abundance and accessibility by means of minimally invasive harvesting procedures. ${ }^{3,4}$ Adipose-derived stem cells (ADSCs) were first investigated as an alternative source of MSCs and considered as most preferred cell type, based on their phenotype, plasticity, and immunomodulatory properties for applications in tissue engineering and regenerative medicine., $3 ., 5$

Regenerative therapy was originally based on the use of three-dimensional scaffolds. ${ }^{6}$ Combination of scaffolds, growth factors, and multipotent stem cells are suggested to increase the efficacy of regenerative approaches. ${ }^{7}$ Particularly, the application of a biodegradable scaffold with an appropriate stem cell, with or without the supplementation of growth factors, is the most routinely followed therapeutic method. ${ }^{1}$ Collagen is the most widely used scaffold material, which adapts in cases such as osteochondral defects, connective tissues, adipose tissue, and mammary glands. ${ }^{8}$ In managing the oral mucosal defects, the xenograft, bovine-derived collagen membrane $(0.5 \mathrm{~mm})$ has proven to be more convenient in its usage. ${ }^{9,10}$ In a recent study, collagen promoted adipocytogenesis, and as a major constituent of extracellular matrix, it provided mechanical rigidity; also, fibrils acted as guidance structures for contacting cells. ${ }^{11}$

With advanced production protocols and development in technologies, such as the concentrated platelet-rich plasma (CPRP), a new generation of platelet concentrates have been identified and optimized for a novel kind of fibrin with adhesive property.$^{12}$ Later on, Choukroun et al first described platelet-rich fibrin (PRF) in 2001, widely used in oral and maxillofacial procedures. ${ }^{13}$ The procedure involves centrifugation of autologous blood, and then resuspension of platelets in a small amount of recovered plasma after the removal of erythrocytes and leucocytes. The property of PRF which makes it possible to retain the growth factors and enables cell migration is credited to its three-dimensional structure. ${ }^{14}$ The effective properties, including angiogenesis and adipogenesis, were demonstrated in PRF as critical requirements in tissue repair. ${ }^{15}$

Studies on the role of PRF in promoting cellular and differentiation properties of stem cells are limited. ${ }^{16,17}$ Reports on human stem cells from the apical papilla (SCAPs), advancement in proliferation, migration, and differentiation, was noted with addition of PRF. Further, PRF enhanced the osteo-/odontogenic differentiation of SCAPs by activating the extracellular signal-regulated kinase (ERK) pathway. ${ }^{17}$ The present study hypothesized that collagen gel and PRF, when used as scaffolding materials, would improve the adipogenicity of ADSCs in vitro. Hence, it was aimed to compare the adipogenic differentiation potential of human ADSCs cultured individually with collagen gel and PRF in vitro.

\section{Materials and Methods}

\section{Ethics Statement}

This study was conducted in accordance to the Declaration of Helsinki principles. The necessary approval was obtained from Institutional Ethics Committee and Institutional Committee for Stem Cell Research (IC-SCR).

\section{Isolation and Culture of ADSCs}

Adipose tissue was collected by liposuction from healthy individuals after obtaining their written consent. The tissue was enzymatically digested using $0.1 \%$ collagenase (Gibco-Invitrogen, Life Technologies, Grand Island, NY, USA) for 2 hours in a humidified incubator. Then, Dulbecco's modified Eagle's medium (DMEM)-High glucose (HG) along with $10 \%$ fetal bovine serum (FBS, Gibco-Invitrogen) was added to neutralize the traces of the enzyme. Debris were
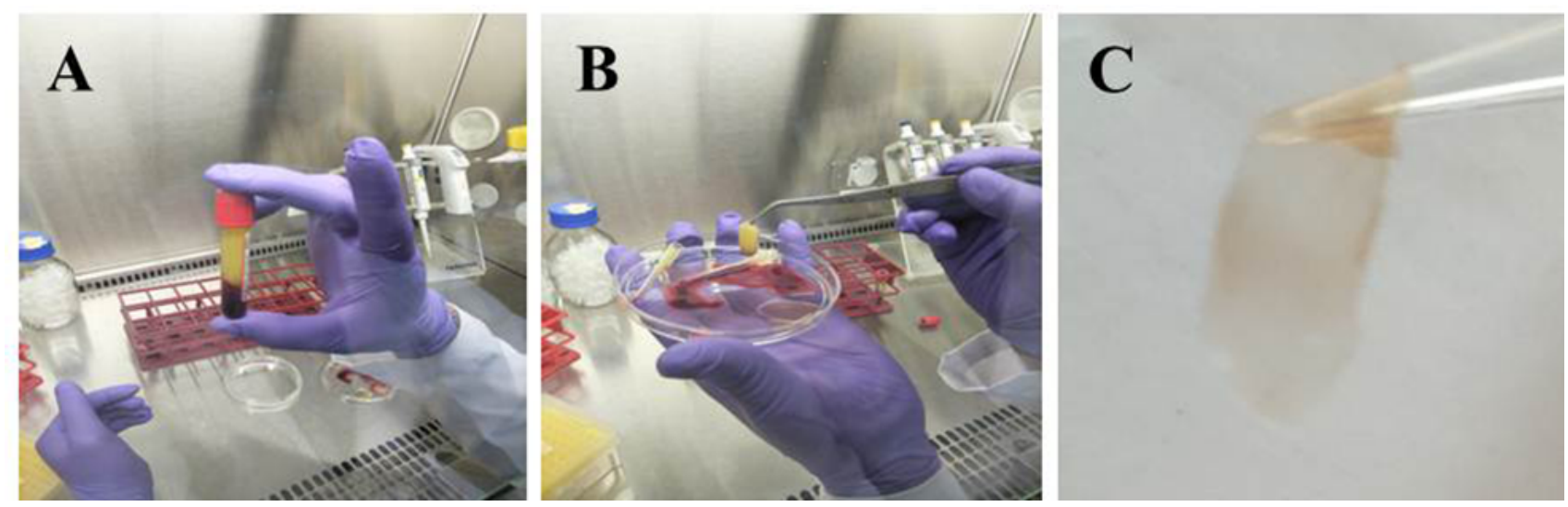

Fig. 1 Preparation of platelet-rich fibrin (PRF). (A). Separation of three layers after centrifugation at $3000 \mathrm{rpm}$ for $10 \mathrm{~min}$. (B). Separation of fibrin from the plasma and RBCs. (C). PRF ready for use or storage. 
removed, and suspended cells were cultured in DMEM-HG with $10 \%$ FBS, $100 \mathrm{U} / \mathrm{mL}$ penicillin and $100 \mu \mathrm{g} / \mathrm{mL}$ streptomycin (Gibco-Invitrogen) at $37{ }^{\circ} \mathrm{C}$ in $5 \% \mathrm{CO}_{2}$ incubator with medium change twice a week. At $80 \%$ confluency, the ADSCs were harvested using $0.25 \%$ trypsin-EDTA (Gibco-Invitrogen) for subpassaging. ADSCs from passage 3 to passage 6 (P3 to P6) were used for all the assays.

\section{Preparation of Collagen Gel and PRF}

Commercially available collagen gel (PureCol EZ Gel- Collagen solution by Biogenuix, New Delhi, India) was used by preparing a firm gel after warming to $37^{\circ} \mathrm{C}$ in an incubator.

PRF was prepared from the peripheral blood sample $(5 \mathrm{~mL})$ collected as a part of craniofacial treatment procedure by following Choukron's protocol. ${ }^{13}$ Briefly, a single spin of blood sample at $3000 \mathrm{rpm}$ for 10 minutes produced three layers, comprising platelet-poor plasma (PPP) in the first layer, PRF in the middle layer, and the bottom layer consisting of erythrocytes ( - Fig. 1 A-C). Harvested PRF was stored in - 80 ${ }^{\circ} \mathrm{C}$ deep freezer (Eppendorf, Hamburg, Germany).

\section{Assessment of Morphology and Viability}

The morphology of ADSCs was assessed at different passages by phase contrast microscopy (Olympus, Tokyo, Japan). Viability of ADSCs was determined by trypan blue $(0.4 \%$, Gibco-Invitrogen) exclusion test using hemocytometer.

\section{Proliferation and Population Doubling Time (PDT) Assay}

ADSCs were seeded at $1 \times 10^{4}$ cells/well in a 12 -well tissue culture plate (Thermo Fisher Scientific, USA) in triplicates. At every three-day interval until day 12 , cells were harvested and counted by hemocytometer. PDT was calculated using a standard formula.
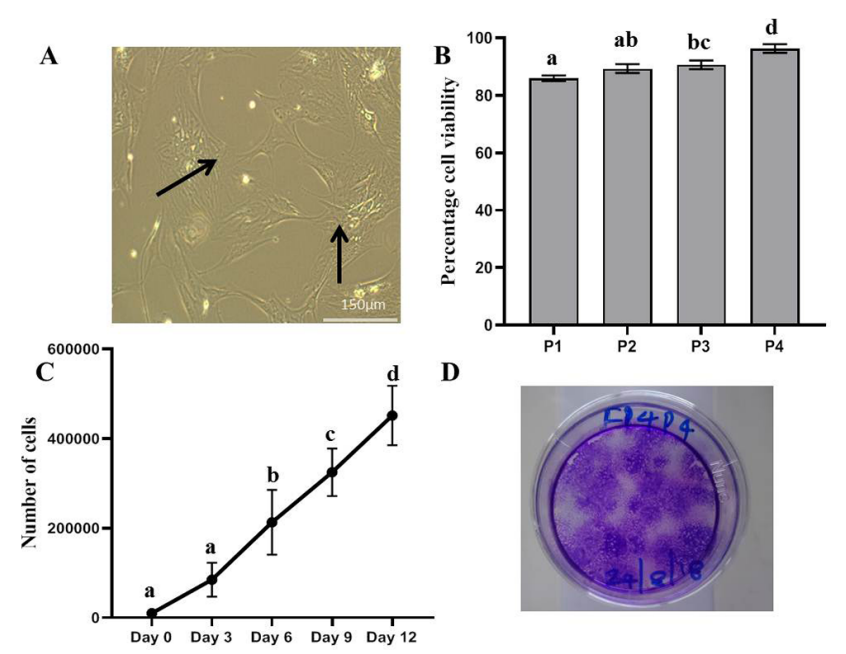

Fig. 2 (A). Isolated adipose-derived stem cells (ADSCs) showed long, sender, fibroblast-like morphology (arrows). (B). The percentage of viability ranged from $86 \%$ to $96 \%$ from passage 1 (P1) to P4. (C). Increase in proliferation rate from day 3 to day 12 was observed in established ADSCs. (D). ADSCs showed the ability to form colonies as stained by crystal violet staining. Superscripts a, b, c, and d indicate statistically significant difference at $p<0.05$.

\section{Colony-Forming Unit (CFU) Assay}

ADSCs were cultured for a period of 14 days at $37{ }^{\circ} \mathrm{C}$ in a $5 \% \mathrm{CO}_{2}$. The cells were stained with $0.1 \%$ crystal violet (Sigma-Aldrich, St. Louis, MO, USA) and incubated at room temperature for 15 minutes. The staining solution was decanted, and approximately 50 cells were considered as a single colony.

\section{Flow Cytometry Analysis}

ADSCs were stained with antibodies against CD29, CD44, CD73, CD90, CD34 and CD45 (eBioscience, CA, USA, or Biolegend, CA, USA). Later, the cells were incubated with fluorescein isothiocyanate (FITC)-conjugated secondary antibody (eBioscience). A total of 10,000 cells were analyzed with flow cytometer (Becton Dickinson, NJ, USA).

\section{Osteogenic Differentiation}

ADSCs were seeded at a density of $1 \times 10^{4}$ cells/well onto 12-well plates and allowed for 21 days in osteogenic induction medium consisting of DMEM-HG supplemented with $0.1 \mu \mathrm{M}$ dexamethasone, $10 \mathrm{mM} \beta$-glycerol phosphate, and $0.2 \mathrm{mM}$ ascorbic acid (Sigma-Aldrich). After induction, cells were stained with Alizarin red (Sigma-Aldrich) for confirming the mineralization.

\section{Adipogenic Differentiation}

ADSCs were seeded at a density of $1 \times 10^{4}$ cells/well onto 12-well plates and cultured for 21 days in adipogenic induction medium containing $0.5 \mu \mathrm{M}$ dexamethasone, $0.5 \mathrm{mM}$ isobutyl methylxanthine, and $50 \mu \mathrm{M}$ indomethacin (Sigma-Aldrich). Adipogenesis was assessed by culturing cells on collagen gel and PRF, individually. ADSCs cultured in basal medium served as control. Assessment of the lipid droplets was made by Oil red O (Sigma-Aldrich) staining.

\section{Statistical Analysis}

Data were expressed as the mean \pm standard deviation (SD) from minimum triplicates. One way-analysis of variance (ANOVA) was carried out by GraphPad Prism software (GraphPad, CA, USA), followed by Tukey's multiple comparison test. Level of significance was set at $p<0.05$.

\section{Results}

\section{Establishment, Morphology and Viability of ADSCs}

The ADSCs were successfully established in vitro. Isolated ADSCs exhibited fibroblast-like characteristics and the cells showed adherence to plastic dishes, which is a property of MSCs (-Fig. 2 A). Trypan-blue exclusion assay showed the viability values ranging from $86 \%$ to $96 \%$ from passage 1 (P1) to P4 ( - Fig. 2 B). Higher viability of ADSCs at later passages indicated that culture conditions did not affect the number of live cells.

\section{Proliferation, PDT and CFU Assay}

Proliferation rate and PDT of ADSCs was analyzed under in vitro conditions. A significant $(p<0.05)$ increase in 

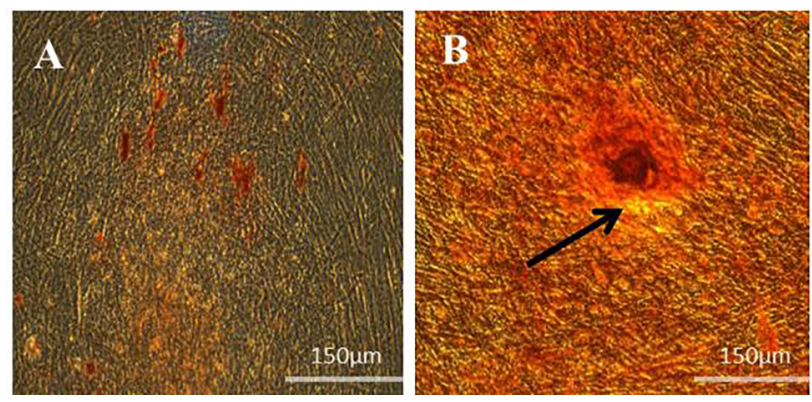

Fig. 3 Osteogenic differentiation of adipose-derived stem cells (ADSCs). (A). ADSCs without osteogenic induction media showing fibroblast-like morphology after 21 days of culture. (B). ADSCs cultured with osteogenic induction media showed the deposition of calcium mineralized nodules (arrow), as evidenced by Alizarin Red $S$ staining.
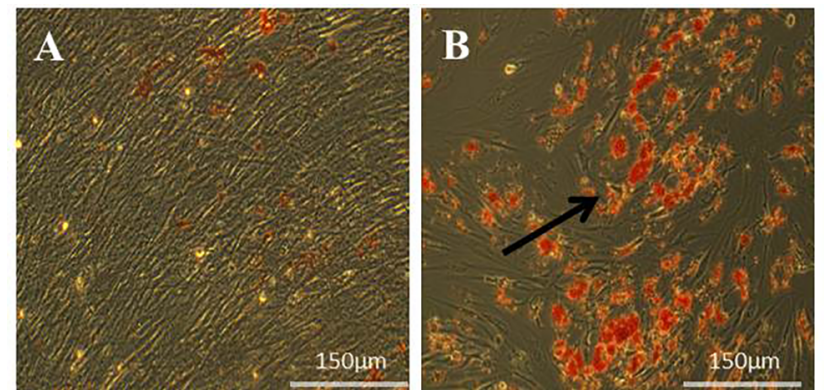

Fig. 4 Adipogenic differentiation of adipose-derived stem cells (ADSCs). (A). ADSCs without adipogenic induction media retained fibroblast-like morphology after 21 days of culture. (B). ADSCs cultured with adipogenic induction media showing the enhanced secretion of neutral lipid vacuoles (arrow), as evidenced by Oil red O staining.
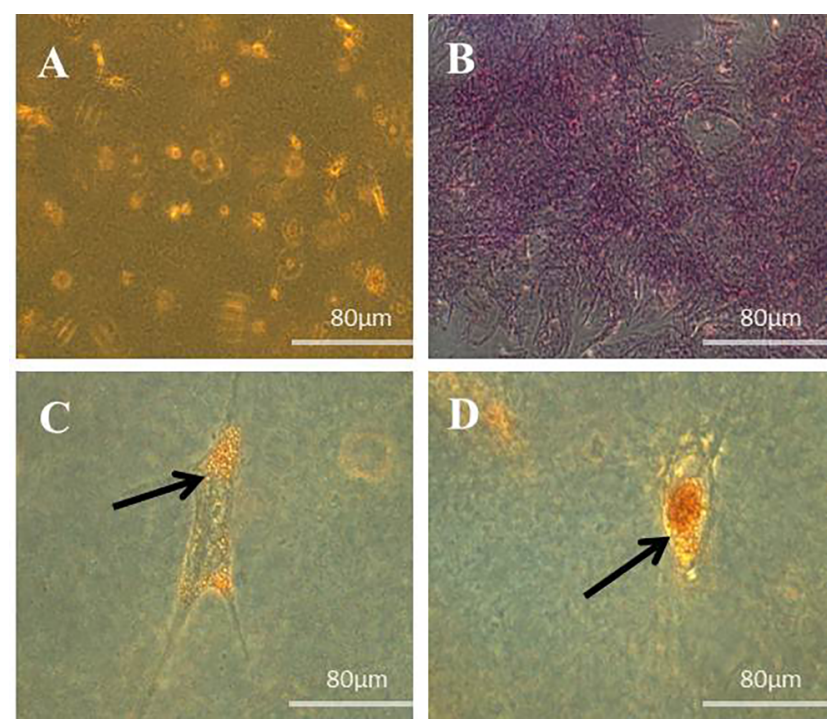

Fig. 5 Adipogenic differentiation of adipose-derived stem cells (ADSCs) cultured on collagen gel. (A). Morphology was slightly flattened on day 3 of culture. (B). Presence of proliferating cells was confirmed by crystal violet staining. (C and $\mathbf{D}$ ). Differentiated adipocytes were indicated by the lipid droplets (arrows), as evidenced by Oil red $\mathrm{O}$ staining.
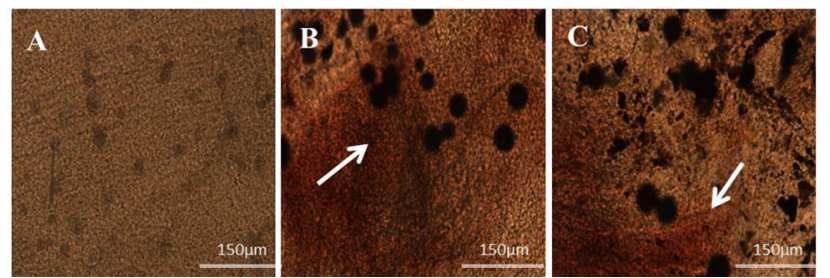

Fig. 6 Adipogenic differentiation of adipose-derived stem cells (ADSCs) cultured on platelet-rich fibrin (PRF). (A). Cells cultured on PRF in control. (B and C). ADSCs stained with Oil red $\mathrm{O}$ solution indicating the presence of neutral lipid droplets (arrows) after 21 days of adipogenic induction.

proliferation rate from day 3 to day 12 was observed in established ADSCs ( - Fig. 2 C). As the time of culture increased, higher the PDT of cells was observed. Further, ADSCs showed the ability to form colonies or proliferate in cluster, although fibroblast-like morphology persisted until reaching the confluence ( - Fig. 2 D).

\section{Flow Cytometry Analysis}

Flow cytometry analysis showed that ADSCs were positive for MSC-specific markers (CD29, CD44, CD73 and CD90) at higher levels (> 80\%) and hematopoietic cell markers (CD34 and CD45) at very low levels ( $<3 \%$ ).

\section{Osteogenic Differentiation}

Osteogenic differentiation of ADSCs was investigated by culturing in specific induction medium for 21 days. ADSCs grown in basal media showed fibroblast-like morphology ( - Fig. 3 A). However, ADSCs cultured with osteogenic induction media for 21 days clearly exhibited the deposition of calcium mineralized nodules, as shown by Alizarin red $\mathrm{S}$ staining (-Fig. $3 \mathbf{B}$ ).

\section{Adipogenic Differentiation}

ADSCs without adipogenic induction media retained their fibroblast-like morphology after 21 days of culture $(\boldsymbol{\sim}$ Fig. 4 A). Oil red $\mathrm{O}$ staining suggested that ADSCs induced with adipogenic differentiation media exhibited the enhanced secretion or synthesis of neutral lipid vacuoles ( - Fig. 4 B).

\section{Adipogenic Differentiation of ADSCs Cultured on Collagen Gel}

Culture of ADSCs on collagen gel showed the adhesion and proliferation activity by day 3 , with slightly flattened morphology (-Fig. 5 A). The number of cells was higher with enhanced proliferation on collagen gel, as stained by crystal violet ( $\boldsymbol{- \text { Fig. }} \mathbf{5}$ B). After adipogenic induction for 21 days, ADSCs differentiated into adipocytes at slightly higher intensity, as visualized by Oil red $O$ staining ( - Figs. 5C and 5D).

\section{Adipogenic Differentiation of ADSCs Cultured on PRF}

Adhesion and proliferation of ADSCs on PRF membrane are presented in - Fig. 6 A. No adipogenic activity was observed in cells cultured with basal medium. However, Oil red $\mathrm{O}$ 
staining (-Fig. 6B and 6C) revealed that ADSCs cultured on PRF with adipogenic induction medium for 21 days had the capability of forming adipocytes at marginally increased intensity.

\section{Discussion}

The array of therapeutic interventions ranges from the treatments that selectively start-up with wound into healing cascade to various other methods that mechanically protect the wound or increase perfusion and oxygenation of the local tissues. ${ }^{18}$ The concept of adipose tissue transplant as filler has evolved of late, and it is presently regarded as a source of abundant MSCs. In this view, studies on the application of ADSCs in head and neck soft-tissue defects have swiftly increased recently. However, there are unanswered questions that limit the clinical translation of ADSCs for craniofacial applications. Hence, this in vitro study assessed the biological properties of ADSCs, and later evaluated their adipogenesis with collagen gel and PRF individually, as a preliminary attempt toward soft-tissue augmentation.

In 2001, Zuk et al analyzed the fundamental cell types and multilineage potential of mesodermal stem cells obtained by enzymatic digestion of lipoaspirates. ${ }^{4}$ In this study, enzymatic digestion was performed for liberating the cells and stromal vascular fraction (SVFs) in the same method. Processing of the lipoaspirates using an enzyme resulted in the successful establishment of ADSCs that exhibited a long, slender, fibroblast-like morphology and showed greater adherence to plastic dishes, confirming the properties of MSCs.

It is essential to evaluate the influence of long-term culture on the phenotype and differentiation potential of ADSCs. Higher viability of ADSCs was observed in the present study, even at passage 6 , indicating that culture conditions did not affect the number of live cells. Cell growth in the presence of biomaterials was assessed, and the observations showed more than 90\% confluency on collagen gel and PRF. The cell viability when cultured on collagen gel and PRF was higher, but not clear due to difficulty in visualization through phase-contrast microscope. Supporting these observations, Girandon et al recorded greater cell viability cultured on the scaffolds. ${ }^{19}$ An earlier study also noticed increased cell viability when ADSCs were held to the scaffold matrix. ${ }^{20}$

The scaffolds used in tissue engineering and regenerative medicine have a specific morphology and surface chemistry, and thereby have a vital effect on behaviour of cells such as adhesion, proliferation, differentiation, and cell-matrix interaction. ${ }^{21}$ In this study, higher adhesion of ADSCs was recorded along with enhanced proliferative potential. However, PDT was longer as the number of cell culture days extended. Further, ADSCs showed the ability to form colonies, indicating their potency characteristics.

The flow cytometry assay signified the positivity of CD29, CD44, CD73 and CD90 markers with no expression of markers for hematopoietic lineage in the cultured ADSCs. For the validation of the purity and potency of ADSCs, surface marker analysis is crucial. ${ }^{22}$ Earlier reports showed the expression of MSC-specific markers in purified ADSCs, such as CD49f, CD44, CD90, CD105, CD13 and CD71. ${ }^{23}$ Further, ADSCs cultured in osteogenic induction media exhibited calcified mineralized nodule deposition, as observed by Alizarin Red S staining. Previous studies have also demonstrated the successful osteogenic differentiation of ADSCs. ${ }^{12,23}$

As observed earlier, adipocyte induction of ADSCs by appropriate treatment resulted in the accumulation of lipid droplets. ${ }^{12,23}$ When ADSCs were coalesced with collagen gel, they exhibited higher cell number and reached $90 \%$ confluency. Bovine collagen gel was selected in this study, as bovine collagen matrix precipitates in wound healing with almost negligible side effects. We observed that ADSCs on collagen gel at confluency stage showed better characteristics of cell viability, adherence, morphology, proliferation capacity, and enhanced adipogenic differentiation, as evidenced by the intensity of Oil red $\mathrm{O}$ staining.

Interestingly, a second generation of platelet concentrate, PRF, forms a 3D matrix and contains all the constituents required for tissue repair. PRF consists of higher concentration of blood derived growth factors and is easier to produce, as it does not require any additives with less centrifugation time, as compared with other similar concentrates. ${ }^{14}$ In this study, ADSCs seeded on to PRF showed moderate compatibility and capability of differentiating into adipocytes at marginally increased intensity. Previously, PRF released autologous growth factors and showed a long-lasting effect on proliferation and differentiation of rat osteoblasts than PRP. ${ }^{24}$ However, studies supporting the potency of PRF in promoting adipogenicity of MSCs are lacking. The quick, effective, and undemanding procedure made us include PRF as one of the biomaterials in this study, and the results indicated that it had moderate influence on adipogenic potential of ADSCs.

\section{Conclusion}

In summary, ADSCs cultured on collagen gel and PRF, individually, showed higher number of differentiated adipocytes than ADSCs grown with induction medium alone. Among the biomaterials, the extent of lipid accumulation was slightly higher on collagen gel than on PRF, since all the intracellular space was occupied with large red-stained lipid vacuoles. However, qualitative assessment alone in this study restricts the definite conclusion, and further quantitative molecular assays are required to confirm better suitability of scaffold for soft-tissue regeneration.

\section{Source of Support}

Nil.

\section{Conflict of Interest}

Authors declare that there are no conflicts of interest.

\section{References}

1 Brett E, Chung N, Leavitt WT, Momeni A, Longaker MT, Wan DC. A review of cell-based strategies for soft tissue reconstruction. Tissue Eng Part B Rev 2017;23(4):336-346

2 Prentice DA. Adult stem cells. Circ Res 2019;124(6):837-839 
3 Conese M, Annacontini L, Carbone A, et al. The role of adipose-derived stem cells, dermal regenerative templates, and platelet-rich plasma in tissue engineering-based treatments of chronic skin wounds. Stem Cells Int 2020;2020:7056261

4 Zuk PA, Zhu M, Mizuno H, et al. Multilineage cells from human adipose tissue: implications for cell-based therapies. Tissue Eng 2001;7(2):211-228

5 Naderi N, Griffin MF, Mosahebi A, Butler PE, Seifalian AM. Adipose derived stem cells and platelet rich plasma improve the tissue integration and angiogenesis of biodegradable scaffolds for soft tissue regeneration. Mol Biol Rep 2020;47(3):2005-2013

6 Egusa H, Sonoyama W, Nishimura M, Atsuta I, Akiyama K. Stem cells in dentistry-Part II: Clinical applications. J Prosthodont Res 2012;56(4):229-248

7 Ishihara M, Kishimoto S, Nakamura S, Fukuda K, Sato Y, Hattori H. Biomaterials as cell carriers for augmentation of adipose tissue-derived stromal cell transplantation. Biomed Mater Eng 2018;29(5):567-585

8 Zhang D, Wu X, Chen J, Lin K. The development of collagen based composite scaffolds for bone regeneration. Bioact Mater 2017;3(1):129-138

9 Howard D, Buttery LD, Shakesheff KM, Roberts SJ. Tissue engineering: strategies, stem cells and scaffolds. J Anat 2008;213(1):66-72

10 Pawelec KM, Best SM, Cameron RE. Collagen: a network for regenerative medicine. J Mater Chem B Mater Biol Med 2016;4(40):6484-6496

11 Zöller N, Schreiner S, Petry L, et al. Collagen I promotes adipocytogenesis in adipose-derived stem cells in vitro. Cells 2019;8(4):302

12 Dohan DM, Choukroun J, Diss A, et al. Platelet-rich fibrin (PRF): a second-generation platelet concentrate. Part I: technological concepts and evolution. Oral Surg Oral Med Oral Pathol Oral Radiol Endod 2006;101(3):e37-e44

13 Choukroun J, Adda F, Schoeffler C, Vervelle A. Une opportunite' en paro-implantologie: Le PRF. Implantodontie 2000;42:55-62

14 Naik B, Karunakar P, Jayadev M, Marshal VR. Role of platelet rich fibrin in wound healing: a critical review. J Conserv Dent 2013;16(4):284-293
15 Liao HT, Marra KG, Rubin JP. Application of platelet-rich plasma and platelet-rich fibrin in fat grafting: basic science and literature review. Tissue Eng Part B Rev 2014;20(4):267-276

16 Hong S, Chen W, Jiang B. Comparative evaluation of concentrated growth factor and platelet-rich fibrin on the proliferation, migration, and differentiation of human stem cells of the apical papilla. J Endod 2018;44(6):977-983

17 Bi J, Liu Y, Liu XM, Lei S, Chen X. Platelet-rich fibrin improves the osteo-/odontogenic differentiation of stem cells from apical papilla via the extracellular signal-regulated protein kinase signaling pathway. J Endod 2020;46(5):648-654

18 Miloro M, Ghali GE, Larsen PE, Waite PD, Chapter 1 Wound healing. In: Shetty V, Bertolami CN, eds. Peterson's Principles of Oral and Maxillofacial Surgery. 2. Hamilton: BC Decker; 2004

19 Girandon L, Kregar-Velikonja N, Božikov K, Barlič A. In vitro models for adipose tissue engineering with adipose-derived stem cells using different scaffolds of natural origin. Folia Biol (Praha 2011;57(2):47-56

20 Flynn L, Prestwich GD, Semple JL, Woodhouse KA. Adipose tissue engineering with naturally derived scaffolds and adipose-derived stem cells. Biomaterials 2007;28(26):3834-3842

21 Mota A, Sahebghadam Lotfi A, Barzin J, et al. Human bone marrow mesenchymal stem cell behaviors on PCL/gelatin nanofibrous scaffolds modified with a collagen IV-derived RGD-containing peptide. Cell J 2014;16(1):1-10

22 Mildmay-White A, Khan W. Cell surface markers on adipose-derived stem cells: A systematic review. Curr Stem Cell Res Ther 2017;12(6):484-492

23 Folgiero V, Migliano E, Tedesco $\mathrm{M}$, et al. Purification and characterization of adipose-derived stem cells from patients with lipoaspirate transplant. Cell Transplant 2010;19(10):1225-1235

24 He L, Lin Y, Hu X, Zhang Y, Wu H. A comparative study of platelet-rich fibrin (PRF) and platelet-rich plasma (PRP) on the effect of proliferation and differentiation of rat osteoblasts in vitro. Oral Surg Oral Med Oral Pathol Oral Radiol Endod 2009;108(5):707-713 